\title{
EFEITOS DOS TERMOS DE TROCA EXTERNOS SOBRE OS PRECOS E A PRODUÇÃO DOMÉSTICA: UMA ANÁLISE DE EQUILÍBRIO GERAL"
}

\author{
Alvaro Barrantes Hidalgo**
}

\section{ABSTRACT}

This work analyzes how domestic prices and production react to changes in international terms of trade, subsidies and incentives to production of exportable goods. Previous works have dealt with some of. these effects upon the Brazilian economy, but their focus was restricted to the interaction between export agriculture and agriculture destined for the domestic market. Here we also take into account interactions with the industrial sector by using a general equilibrium model. In section two, we build a theoretical model, showing that the effect of a change in the international terms of trade upon the real price of domestic goods depends only on the ranking of factor intensities on the sectors to be conside red. An improvement in the international terms of trade lead to an increase in the real price of the domestic good only when the industrial sector is more intensive in capital (or labor) than the

* 0 presente trabalho originou-se dos capitulos 2 e 3 da Tese de Doutorado do autor, apresentada à Universidade de São Paulo (Barrantes, 1983). Agradeço os comentários e sugestões recebidos do meu orientador, Professor Maurício Barata de Paula Pinto. Os erros e omissōes remanescentes são, naturalmente, de minha responsabilidade. Agradeço o apoio $\mathrm{f}$ inanceiro recebido do CNPq.

** 0 autor é Professor e Pesquisador do Programa de Pös-Graduação em Economia (P MES) do Departamento de Economia da UFPE. 
three sectors considered. In section three we show that the effect of a change in the international terms of trade on the quanti ty of goods produced does not depend only on factorial intensities but also on consumer preference. An improvement in the terms of trade will always increase the production of exportable goods and decrease the production of domestic goods, as long as we admit that both goods are gross complements (or independent) that the damestic good is inferior, and that the import sector is more intensive in capital (or labor) than the three sectors of the economy. 
RESUMO

Este trabalho tem por objetivo analisar como os preços e a produção de bens no mercado interno reagem às alterações nos termos de troca do comércio internacional, aos subsídios e incentivos à produção de exportáveis. Trabalhos anteriores examinaram alguns desses efeitos considerando apenas a interaçāo entre a agricultura de exportação e a agricultura voltada para o mercado interno. Neste trabalho utilizamos um modelo de equilíbrio geral, onde se leva em conta também as interrelações com o setor produtor de bens subs titutos de importāveis. Na Seção 2 construímos o modelo teórico e mostramos que o efeito de uma modificação nos termos de troca do comércio internacional, sobre o preço real do bem doméstico, depen de apenas da ordenação das intensidades fatoriais vigorando em cada um dos setores em que foi dividida a economia. Uma melhoria nos termos de troca externos leva a um aumento no preço real do bem do méstico somente quando o setor produtor de bens substitutos de importáveis é o mais intensivo em capital (trabalho) dos três seto res considerados. Na seção 3 mostramos que o efeito de uma mudança nos termos de troca externọs sobre a quantidade produzida de bens depende não apenas das intensidades fatoriais, mas também das preferências dos consumidores. Uma melhoria nos termos de troca sem pre aumentará a produção do bem exportável e diminuirá a produçāo do bem doméstico, desde que admitamos que o bem doméstico e o bem exportável são complementares brutos (ou independentes), sendo o bem doméstico inferior e o setor importador o mais intensivo em ca pital (trabalho) dos três setores da economia. 



\section{INTRODUCRAO}

Em meados da década de 1960, vārias economias menos desenvolvidas começaram a mudar suas estratégias de crescimento econômico. o setor exportador, que tinha caído em um processo de estagnação nos anos anteriores, passou a desempenhar um papel fundamental den tro da nova estratégia. Tendo em mente esta preocupação, diversos instrumentos de politicas foram usados. Os esquemas de controles sobre o comércio exterior foram abrandados e parcialmente substituídos por politicas mais liberais. Criaram-se subsidios e créditos à exportação e introduziram-se sistemas cambiais mais realistas. Esperava-se que tais medidas fossem capazes de expandir as receitas de exportações. Para ter sucesso, a nova política tinha que alterar os preços locais dos bens exportáveis, em relação aos outros preços domésticos, incentivando assim a produção de exportá veis.

Com os novos incentivos, esperava-se uma resposta positiva na produção doméstica desses bens. Naturalmente, o sucesso dessa nova política dependia das elasticidades da demanda internacional dos bens exportáveis.

Após alguns anos de experiência com o novo sistema $e$, tendo em vista, as boas condições no mercado internacional, as exportações de alguns países menos desenvolvidos realmente cresceram a taxas razoavelmente altas. Desse ponto de vista, a reorientação da politica comercial parece ter sido bem sucedida.

Entretanto, o novo sistema tem experimentado algumas dificuldades para alterar os preços relativos dos bens no mercado doméstí co. Os incentivos especificos ao setor exportador, os créditos con cedidos às exportações e a nova política cambial parecem ter exercîo um impacto inflacionário sobre os preços dos bens não comer- 
ciáveis. No caso do Brasil, alguns desses fenômenos parecem ter acontecido. Alguns autores, preocupados com os efeitos da politica de promoção das exportações brasileiras, concluem, por exemplo, que parte dos beneficios do esforço exportador estão sendo anulados pe la queda na produção de bens domésticos (basicamente alimentos), e pela correspondente elevação de seus preços. Argumenta-se que a maior rentabilidade do setor exportador, quando comparada com o se tor de bens não-comerciáveis, e devida tanto a desvalorizações cam biais mais frequentes como a subsídios e créditos à exportação ou a melhores condições nos mercados internacionais, tem afetado seriamente a produção de bens para o mercado doméstico (1).

Por outra parte, em um contexto de longo prazo, a abertura ao exterior parece ter induzido um maior grau de tecnificação no setor exportador, aumentando em consequência o diferencial de modernização entre os setores comerciáveis e de produtos domésticos. Co mo consequência de tudo isso, argumenta-se, a produção no setor ex portador tem-se expandido nos últimos anos. Por outro lado, a ofer ta e a demanda de bens domésticos são uma função apenas das condições internas de oferta e demanda. A expansão do setor exportador, empregando cada vez maiores quantidades de insumos, pressiona o mercado de fatores, elevando seus preços. Assim, dada a dotação de insumos na economia, o custo de produção dos bens domésticos também se eleva e, portanto, as quantidades originais só serão oferecidas a preços maiores. Esta pressão para cima dos preços dos alimentos, argumenta-se, tem sérias consequências sobre a renda e bem -estar dos assalariados urbanos.

o objetivo deste trabalho é construir um modelo teórico, for-

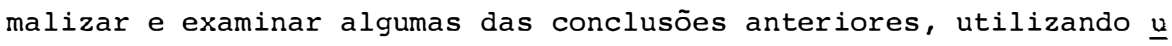
ma estrutura de análise de equilíbrio geral. Tendo em vista que o estudo de alguns desses problemas tem sido feito partindo de mode los de equilíbrio parcial (2), ficando, portanto, dificil examinar os efeitos de mudanças nos termos de troca entre produtos agricolas e industriais, trabalha-se aqui com um modelo mais desagregado, onde um terceiro setor, produtor de bens substitutos de importáveis, è considerado explicitamente. 
Na Seção 2 deste trabalho formulamos o modelo e examinamos o efeito de alterações nos termos de troca internacionais ou nos incentivos ou subsídios à exportação, sobre o preço real dos bens do mésticos. Mostraremos que a direçāo desse efeito depende apenas da ordenação das intensidades fatoriais, nos setores considerados. Veremos que essa condição independe de estarmos trabalhando com coe ficientes de produção fixos ou variāveis. Na seção 3 analisamos o efeito de mudanças favoráveis, nos termos de troca externos, sobre a quantidade produzida de bens domésticos e comerciáveis. Mostrare mos que esse efeito depende não apenas da ordenação das intensidades fatoriais, mas também das preferēncias dos consumidores. Na Se ção 4 apresentaremos as principais conclusões do trabalho. Nos Apēndices 1 e 2 ampliaremos o modelo e examinaremos algumas variáveis de longo prazo. No Apēndice 1 analisaremos o efeito de mudanças exógenas nas dotações de fatores de produção sobre o nível de produção de bens domésticos e comerciāveis. No Apēndice 2 incorporamos o progresso técnico à anālise.

\section{A INTERACÁO ENTRE D SETOR EXTERNO E 0 SETOR DOMESTICO: $O$ MDDELO BASSICD}

Considere-se uma economia pequena (tomando os preços internacionais como dados) em um determinado instante de tempo, produzindo e consumindo três classes de bens: um primeiro bem exportável $\mathrm{x}_{1}$; um segundo bem $\mathrm{x}_{2}$ substituto dos importáveis; e um terceiro bem $\mathrm{x}_{\mathrm{N}}$ não-comerciável, com custos de transporte proibitivos entre paises e custos de transporte nulos dentro das fronteiras do país. A justificação para considerar explicitamente bens nāo-comerciāveis na análise dos problemas do comércio internacional deriva de que, em qualquer economia, uma grande proporção do produto nacional está formada por bens que não conseguem participar dos fluxos do co mércio. Isto devido não somente à existēncia de custos de transpor te, como apontado acima, mas também devido à existência de tarifas alfandegārias, restrições comerciais, ou gostos dos consumidores marcadamente diferentes. 
Na produção dos bens $x_{i}$, os empresários usam dois fatores de produção: trabalho (L) e capital (K), cuja dotação consideramos fi $\mathrm{xa}^{(3)}$. Postula-se que a demanda agregada por cada bem é obtida a partir do somatōrio horizontal das demandas individuais, as quais se admite que existam.

Os excessos de demanda, para cada mercadoria, são expressos como:

$$
E_{i}=D_{i}-x_{i} \quad i=1,2, N
$$

sendo $D_{i}$ e $x_{i}$ as funções de demanda e oferta agregada pelo bem $i$, respectivamente. As demandas são funções dos preços dos bens, $\mathrm{P}_{i}$ ' expressos em termos de um numerário arbitrārio e do nível da renda doméstica, $\mathrm{Y}$, definida como:

$$
\mathrm{Y}=\mathrm{X}_{1} \mathrm{P}_{1}+\mathrm{X}_{2} \mathrm{P}_{2}+\mathrm{X}_{\mathrm{N}} \mathrm{P}_{\mathrm{N}}
$$

Podemos escrever:

$$
D_{i}=D_{i}\left[P_{1}, P_{2}, P_{N}, Y\left(P_{1}, P_{2}, P_{N}\right)\right]
$$

Os excessos de demanda são negativos para o bem 1 , positivos para o bem 2 e zero para o bem doméstico. Este último significa que em equilíbrio a demanda do bem doméstico é igual à oferta; esta con dição permite que o sistema de trēs bens e dois fatores possa ter solução(4).

Tem-se então:

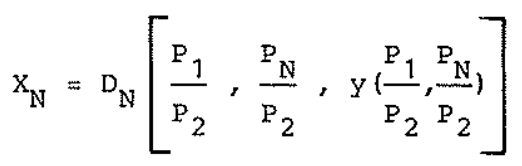

sendo $\mathrm{y} \circ$ nível da renda real doméstica, $\mathrm{P}_{1} / \mathrm{P}_{2}$ e $\mathrm{P}_{\mathrm{N}} / \mathrm{P}_{2}$, os preços relativos dos bens exportáveis e bens domésticos, respectivamente, e todas as variáveis expressas em termos de bens importáveis. Em equilíbrio a seguinte condição para o balanço de pagamentos é obedecida:

$$
\mathrm{P}_{1} \mathrm{E}_{1}+\mathrm{P}_{2} \mathrm{E}_{2}=0
$$


Com relação à tecnologia, neste trabalho vamos analisar os ca sos em que a função de produção apresenta coeficientes fixos e, também, o caso mais geral de coeficientes variāveis de produção (5) Mostraremos que as conclusões desta seção são as mesmas, independentemente das funções de produção apresentarem coeficientes fixos ou variāveis. Se as funções de produção apresentam coeficientes fixos, então a tecnologia da economia pode ser representada por meio da seguinte matriz:

$$
A=\left[\begin{array}{ccc}
\frac{L_{1}}{x_{1}} & \frac{L_{2}}{x_{2}} & \frac{L_{N}}{x_{N}} \\
\frac{K_{1}}{x_{1}} & \frac{K_{2}}{x_{2}} & \frac{K_{N}}{x_{N}}
\end{array}\right]=\left[\begin{array}{lll}
a_{L 1} & a_{L 2} & a_{L N} \\
a_{K 1} & a_{K 2} & a_{K N}
\end{array}\right]
$$

onde cada coluna dessa matriz representa completamente a tecnologia de cada setor. Cada $a_{j i}$ representa a quantidade do insumo $j$ ne cessārio para produzir uma unidade do bem i. E conveniente fazer outras hipóteses para facilitar a análise. Admitamos rendimentos constantes de escala. Então, a demanda total de cada insumo é dada pelo coeficiente de insumo-produto multiplicado pelo nível de produto. Os requisitos para que ambos os insumos sejam utilizados até o nível do pleno emprego são dados pelas seguintes equações:

$$
\begin{aligned}
& a_{L 1} x_{1}+a_{L 2} x_{2}+a_{L N} x_{N}=L \\
& a_{K 1} x_{1}+a_{K 2} x_{2}+a_{K N} x_{N N}=K
\end{aligned}
$$

Admitindo a hipótese de equilíbrio competitivo, os custos uni tários de produção em cada indústria devem ser iguais ao preço de cada bem. podemos representar os custos unitários de produção pelo produto das colunas da matriz A, vezes o preço de cada insumo. Então, se todos os bens são produzidos seus custos médios refletem preços de mercado:

$$
a_{E 1} w+a_{K 1} r=P_{1}
$$




$$
\begin{aligned}
& a_{\mathrm{L} 2} w+a_{K 2} r=\mathrm{P}_{2} \\
& a_{\mathrm{LN}} \mathrm{w}+\mathrm{a}_{\mathrm{KN}} \mathrm{r}=\mathrm{P}_{\mathrm{N}}
\end{aligned}
$$

sendo w a taxa de salário e $r$ o preço dos serviços do capital.

As equaçōes 3 e 5 a 9 formam um sistema de seis equações para serem resolvidas, para também seis variáveis: $x_{1}, x_{2}, x_{N}, w, r$ e $\mathrm{P}_{\mathrm{N}}$. Observe-se que neste tipo de modelo, dados os termos de troca internacionais e com coeficientes fixos de produção, as condiçōes de demanda e as dotaçōes de fatores não têm relevância na determinação dos preços dos bens domésticos ou na determinação da remuneração dos fatores de produção.

A solução do caso mais geral de coeficientes variáveis de prọ dução requer a especificação de seis relaçōes adicionais que deter minem os coeficientes insumo-produto. Estas seis relaçōes podem ser obtidas levando-se em consideração que em equilíbrio competiti vo cada coeficiente insumo-produto depende apenas da relação dos preços dos fatores de produção, ou seja:

$$
a_{j i}=a_{j i}(w / r), \begin{aligned}
& j=L, K \\
& i=1,2, N
\end{aligned}
$$

Diferenciando as equaçōes de (5) a (9) e transformando temos:

$$
\begin{aligned}
& \lambda L_{1} \delta_{1}+\lambda L_{2} \delta_{2}+\lambda L_{N} \delta_{N}+\lambda L_{1} \bar{a}_{L 1}+\lambda L_{2} \bar{a}_{L 2}+\lambda L_{N} \bar{a}_{L N}=\mathrm{L} \\
& \lambda \mathrm{K}_{1} \mathrm{~s}_{1}+\lambda \mathrm{K}_{2} \mathrm{~s}_{2}+\lambda \mathrm{K}_{\mathrm{N}} \mathrm{s}_{\mathrm{N}}+\lambda \mathrm{K}_{1} \overline{\mathrm{a}}_{\mathrm{K} 1}+\lambda \mathrm{K}_{2} \overline{\mathrm{a}}_{\mathrm{K} 2}+\lambda \mathrm{K}_{\mathrm{N}} \overline{\mathrm{a}}_{\mathrm{KN}}=\mathrm{R} \\
& \Theta_{L 1} \bar{w}+\Theta_{L 1} \bar{a}_{L 1}+\Theta_{K 1} \bar{r}+\Theta_{K 1} \bar{a}_{K 1}=\hat{P}_{1} \\
& \ominus_{L 2} \bar{w}+\ominus_{L 2} \bar{a}_{L 2}+\Theta_{K 2} \hat{r}+\Theta_{K 2} \bar{a}_{K 2}=\hat{P}_{2} \\
& \Theta_{L N} \bar{w}+\Theta_{L N} \bar{a}_{L N}+\Theta_{K N} \hat{r}+\Theta_{K N} \hat{a}_{K N}=\hat{P}_{N}
\end{aligned}
$$

onde a notação " -" indica a mudança relativa em cada variável ou parãmetro, por exemplo: 
$\mathrm{S}_{1}=\frac{\mathrm{dx}_{1}}{\mathrm{x}_{1}} ; \mathrm{e} \lambda \mathrm{L}_{i}=\frac{\mathrm{L}_{i}}{\mathrm{~L}}, \lambda \mathrm{K}_{i}=\frac{\mathrm{K}_{i}}{\mathrm{~K}}, \Theta_{\mathrm{L} i}=w \mathrm{~L}_{i} /\left(\mathrm{P}_{i} \mathrm{X}_{i}\right) \quad \mathrm{e}$ $\Theta_{K i}=r \cdot K_{i} /\left(P_{i} x_{i}\right), \quad i=1,2, N$.

Se os coeficientes de produção são fixos, as expressões de (10) a (14) se simplificam sobremaneira, tendo em vista que neste caso particular a taxa de variação do coeficiente insumo-produto é zero. Como foi demonstrado por Jones (1965), no caso geral de coeficientes variáveis de produção, condições adicionais podem ser obtidas e simplificar as expressões acima. Assim, consideremos uma firma típica. Para um dado nível de produção, ela procura minimizar os custos, isto é, minimiza os custos unitários. No setor dois, por exemplo, os custos médios estão dados por:

$$
a_{L 2} \cdot w+a_{K 2} \cdot r
$$

Levando em conta que a firma toma como dados os preços dos insumos, derivando (15), igualando a zero e transformando, chegamos à seguinte expressão:

$$
\Theta_{L 2} \cdot \hat{a}_{L 2}+\theta_{K 2} \cdot \bar{a}_{K 2}=0
$$

Procedimento semelhante pode ser seguido nos setores de bens exportáveis e bens domésticos, chegando-se às seguintes expressões:

$$
\begin{aligned}
& \theta_{L 1} \cdot \hat{a}_{L 1}+\theta_{K 1} \cdot \hat{a}_{K 1}=0 \\
& \theta_{L N} \cdot \hat{a}_{L N}+\theta_{K N} \cdot \hat{a}_{K N}=0
\end{aligned}
$$

Substituindo as equações (16) a (18) nas expressões (12) a (14), podemos simplificar ainda mais e obter as seguintes expressões, que vamos chamar equações de mudanças de preço: .

$$
\begin{aligned}
& \Theta_{L 1} \hat{w}+\theta_{K 1} \hat{r}=P_{1} \\
& \theta_{L 2} \hat{w}+\theta_{K 2} \hat{r}=P_{2} \\
& \Theta_{L N} \hat{w}+\theta_{K N} \hat{r}=P_{N}
\end{aligned}
$$


Fica assim demonstrado que a relação entre mudanças nos preços dos fatores de produção e mudanças nos preços dos bens é a mes ma, independentemente de estarmos trabalhando com coeficientes de produção fixos ou variāveis ${ }^{(6)}$.

Resolvendo as equações $\left(12^{\prime}\right)$ e (13') temos:

$$
\frac{1}{\left|d_{1}\right|}\left(\hat{P}_{1}-\hat{P}_{2}\right)=(\hat{w}-\hat{r})
$$

onde

$$
\left|\mathrm{d}_{1}\right|=\left|\begin{array}{ll}
\theta_{\mathrm{L} 1} & \theta_{\mathrm{K} 1} \\
\theta_{\mathrm{L} 2} & \theta_{\mathrm{K} 2}
\end{array}\right|
$$

De forma semelhante, de $\left(13^{\prime}\right)$ e $\left(14^{\prime}\right)$ temos que:

$$
\frac{1}{\left|d_{2}\right|}\left(\hat{P}_{2}-\hat{P}_{N}\right)=(\hat{w}-\hat{r})
$$

sendo

$$
\left|a_{2}\right|=\left|\begin{array}{ll}
\theta_{L 2} & \theta_{K 2} \\
\theta_{L N} & \theta_{K N}
\end{array}\right|
$$

Dividindo, agora, a equação (19) pela equação (20) e simplificando, tem-se ${ }^{(7)}$ :

$$
\left(\mathrm{P}_{1}-\mathrm{P}_{2}\right)=\left(\mathrm{P}_{\mathrm{N}}-\mathrm{P}_{2}\right) \cdot\left(\frac{\mathrm{k}_{1}-\mathrm{k}_{2}}{\mathrm{k}_{\mathrm{N}}-\mathrm{k}_{2}}\right) \cdot\left(\frac{\mathrm{P}_{\mathrm{N}} / \mathrm{a}_{\mathrm{LN}}}{\mathrm{P}_{1} / \mathrm{a}_{\mathrm{L} 1}}\right)
$$

Sendo $k_{i}=\frac{k_{i}}{I_{i}}$ e $a_{L N}$ e $a_{L 1}$ coeficientes técnicos, como defi nidos àcima. 
Esta expressão servirá de base para fazer a análise dos efei tos sobre os preços. Examinemos, em primeiro lugar, o efeito dos termos de troca externos sobre o preço relâtivo do bem doméstico. o último termo, entre parēnteses, do lado direito dessa expressão, é positivo. Pode-se concluir, então, que um aumento (diminuição), no preço relativo do bem exportável, leva a um aumento (diminuição) no preço relativo do bem doméstico, apenas se as seguintes con dições forem vālidas:

$$
k_{2}=\operatorname{ou}_{\text {minimo }}^{\text {máximo }}\left\{k_{1}, k_{2}, k_{\mathrm{N}}\right\}
$$

Assim, a condição para que o preço relativo do bem doméstico varie na mesma direção dos termos de troca externos é que o bem substituto dos importáveis seja mais intensivo em capital (ou em trabalho), que ambos os outros bens. Por outro lado, um aumento (di minuição) nos termos de troca externos leva a uma diminuição (aumento), do preço relativo do bem doméstico, apenas nos casos em que as seguintes condições forem vālidas:

$$
\begin{aligned}
& \mathrm{k}_{1}>\mathrm{k}_{2}>\mathrm{k}_{\mathrm{N}} \\
& \mathrm{ou} \\
& \mathrm{k}_{\mathrm{N}}>\mathrm{k}_{2}>\mathrm{k}_{1}
\end{aligned}
$$

As conclusões anteriores foram obtidas escolhendo o bem 2 (ben importāvel), como numerārio. Entretanto, considerações semelhantes podem ser feitas admitindo o caso em que o bem exportável (bem 1), é escolhido como numerário. Assim, seguindo um procedimento semelhante chega-se à seguinte expressão:

$$
\left.\left(P_{1}-P_{2}\right)=\left(P_{N}-P_{1}\right) \cdot \frac{k_{2}-k_{1}}{k_{1}-k_{N}}\right) \cdot\left(\frac{P_{N} / a_{L N}}{P_{2} / a_{L 2}}\right)
$$

o terceiro termo do lado direito de (21') é positivo e um aumento (diminuição) nos termos de troca externos leva também a um aumento (diminuição) no preço relativo do bem doméstico (agora expresso em termos de exportáveis), sempre que as seguintes - condições sejam vālidas: 


$$
\begin{aligned}
& k_{2}>k_{1}>k_{N} \\
& \text { ou } \\
& k_{N}>k_{1}>k_{2}
\end{aligned}
$$

Finalmente, um aumento (diminuição) nos termos de troca leva a uma diminuiçã்o (aumento) no preço do bem doméstico, expresso em termos do bem exportável, apenas nos casos em que:

$$
k_{1}=\operatorname{ou}_{\text {minimo }}^{\text {máximo }}\left\{\mathrm{k}_{1}, \mathrm{k}_{2}, \mathrm{k}_{\mathrm{N}}\right\}
$$

Os resultados obtidos anteriormente são apresentados enı forma resumida na seguinte tabela:

\section{Tabela 1}

EFEITOS DE MUDANCAS NOS TERMOS DE TROCA

SOBRE O PRECO RELATIVO DO BEM DOMESTICO: CASOS POSSIVEIS

$$
\left(\mathrm{P}_{\mathrm{N}}-\mathrm{P}_{2}\right)>0 \quad\left(\mathrm{P}_{\mathrm{N}}-\mathrm{P}_{1}\right)>0
$$

$$
\begin{array}{ccc}
\left(\mathrm{F}_{1}-\mathrm{s}_{2}\right)>0 & \mathrm{k}_{\mathrm{N}}<\mathrm{k}_{2}>\mathrm{k}_{1} & \mathrm{k}_{2}>\mathrm{k}_{1}>\mathrm{k}_{\mathrm{N}} \\
& \text { ou } & \text { ou } \\
& \mathrm{k}_{\mathrm{N}}>\mathrm{k}_{2}<\mathrm{k}_{1} & \mathrm{k}_{\mathrm{N}}>\mathrm{k}_{1}>\mathrm{k}_{2} \\
& \mathrm{k}_{1}>\mathrm{k}_{2}>\mathrm{k}_{\mathrm{N}} & \mathrm{k}_{\mathrm{N}}<\mathrm{k}_{1}>\mathrm{k}_{2} \\
\left(\mathrm{P}_{1}-\mathrm{s}_{2}\right)<0 & \text { ou } & \text { ou } \\
& \mathrm{k}_{\mathrm{N}}>\mathrm{k}_{2}>\mathrm{k}_{1} & \mathrm{k}_{2}>\mathrm{k}_{1}<\mathrm{k}_{\mathrm{N}}
\end{array}
$$

A análise dos problemas de economia internacional, quando o número de bens ou de fatores é maior que dois, torna-se pouco intuitiva e a interpretação dos resultados se dificulta. Apesar disso, com base nas expressões (19) e (20), a seguir tentaremos dar 
uma interpretação econômica a esses resultados. A equação (19) expressa o teorema Stolper-Samuelson. Examinemos o efeito de uma melhoria nos termos de troca sobre as remunerações dos fatores, admi tindo que o setor exportador é mais intensivo em trabalho que o se tor importador $\left(k_{2}>k_{1}\right)$.

Em um modelo com dois bens, uma melhoria nos termos de troca expande a produção de bens exportáveis e contrai a produção de importáveis. O método de produção no setor de bens exportāveis torna -se mais capital-intensivo. A intensidade de capital também aumenta no setor produtor de bens importáveis, porque, à medida que começa a se expandir a produção de exportáveis (que são por hipótese trabalho-intensivo), há certo desejo de se conseguir mais traba lhadores, o que leva ao aumento do salário. Os produtores tentam substituir trabalho por capital e os métodos de produção tornam-se mais intensivos em capital. O resultado é que o preço dos serviços do capital cai e o salário aumenta. Este resultado, que expressa o teorema Stolper-Samuelson, mais a expressão (20), podem ajudar-nos a interpretar o primeiro resultado da primeira linha e coluna da ta bela anterior. Se o salārio está aumentando, o preço do bem doméstico expresso em termos de importáveis só poderá aumentar se o bem doméstico for também relativamente mais intensivo em trabalho que o setor importador. Isto é precisamente o que indica o primeiro re sultado da primeira linha e coluna daquela tabela. Raciocínio seme lhante pode-se seguir na interpretação dos demais resultados.

A partir das equações de mudanças de preços, pode-se também analisar os efeitos da criação de um subsídio no setor de bens exportáveis sobre o preço relativo do bem doméstico. Se consideramos $\mathrm{P}_{1}$ como sendo o preço de mercado do bem exportável, então, após a introdução do subsídio, o preço recebido pelo produtor será: s.P 1 , sendo s: 1 mais a taxa de subsídio "ad valorem" sobre exportações. A criação do subsídio modifica a equação de mudança de preço (12'), tendo-se:

$$
\Theta_{L 1} \bar{w}+\theta_{K 1} \hat{r}=\hat{P}_{1}+\hat{s}
$$


Resolvendo de novo as equações (12"), (13') e (14') e mantendo constante o preço de mercado do bem exportável, chega-se à seguinte expressão:

$$
\left(\hat{\mathrm{s}}-\hat{\mathrm{P}}_{2}\right)=\left(\hat{\mathrm{P}}_{N}-\hat{\mathrm{P}}_{2}\right) \cdot\left(\frac{\mathrm{k}_{1}-\mathrm{k}_{2}}{\mathrm{k}_{\mathrm{N}}-\mathrm{k}_{2}}\right) \cdot\left(\frac{\mathrm{P}_{\mathrm{N}} / \mathrm{a}_{L N}}{\mathrm{P}_{1} / \mathrm{a}_{L 1}}\right)
$$

o lado direito das expressões (21) e (21") é o mesmo; portanto, a criação de um subsídio no setor de bens exportáveis, expresso em termos de importáveis, aumenta o preço relativo do bem domés tico somente se as condições (22) fossem vālidas.

Fica assim demonstrado que o efeito de uma modificação nos ter mos de troca externos e (ou) nos incentivos e subsídios à exporta ção sobre o preço real do bem doméstico depende da estrutura tecno lógica vigorando em cada um dos setores em que foi dividida a economia. Saber qual é a ordenação das intensidades fatoriais em vigor na economia brasileira é uma questão empirica. Conforme mostra mos em outro trabalho, a evidência empirica parece indicar que no caso da económia brasileira o setor produtor de bens substitutos de importáveis é o setor relativamente mais intensivo em capital dos três setores considerados ${ }^{(8)}$.

3. MUDANCAS NOS TERMOS DE TROCA E A PRODUÇÃO DE BENS

o estudo dos efeitos de mudanças nos termos de troca sobre a quantidade produzida de bens é feito a partir das equações (10) e (11), obtidas na seção anterior, as quais expressam as mudanças na produção:

$$
\begin{aligned}
& \lambda L_{1} \mathrm{~B}_{1}+\lambda \mathrm{L}_{2} \mathrm{~A}_{2}+\lambda \mathrm{L}_{\mathrm{N}} \mathrm{\Omega}_{\mathrm{N}}=\mathrm{L}-\left(\lambda \mathrm{L}_{1} \overline{\mathrm{a}}_{\mathrm{L} 1}+\lambda \mathrm{I}_{2} \hat{\mathrm{a}}_{\mathrm{L} 2}+\lambda \mathrm{L}_{N} \overline{\mathrm{a}}_{L N}\right) \\
& \lambda \mathrm{K}_{1} \mathrm{~A}_{1}+\lambda \mathrm{K}_{2} \mathrm{x}_{2}+\lambda \mathrm{K}_{\mathrm{N}} \mathrm{Q}_{\mathrm{N}}=\mathrm{R}-\left(\lambda \mathrm{K}_{1} \hat{\mathrm{a}}_{\mathrm{K} 1}+\lambda \mathrm{K}_{2} \hat{\mathrm{a}}_{\mathrm{K} 2}+\lambda \mathrm{K}_{\mathrm{N}} \overrightarrow{\mathrm{a}}_{\mathrm{KN}}\right)
\end{aligned}
$$

o último termo entre parēnteses do lado direito da equação (10) é uma média ponderada de mudanças nos coeficientes insumo- 
-produto, usando como ponderação a proporção de trabalho utilizado em cada setor na dotação total da economia. Interpretação semelhante pode ser feita para a equação. (11). De outra parte temos, também, que as mudanças nas proporções dos fatores utilizados, como consequência de uma mudança nos preços desses insumos, depende da elasticidade de substituição. Considerando uma situação de equi líbrio competitivo, é possivel definir a elasticidade de substitui ção da seguinte forma, como desenvolvido por Jones (1965, p. 560).

$$
\sigma_{i}=\frac{\hat{a}_{K i}-\hat{a}_{L i}}{\hat{w}-\hat{r}} \quad i=1,2, N
$$

As equações (16) a (18) e (24) formam um sistema de seis equa ções em oito variáveis (seis taxas de variação de coeficientes insumo-produto, a mudança da taxa de salários e a mudança do preço dos serviços do capital). Pode-se, então, expressar o efeito de mu danças nos preços dos fatores de produção, sobre os coeficientes in sumo-produto, da seguinte forma:

$$
\begin{aligned}
& \hat{a}_{L i}=-\theta_{K i}{ }^{\sigma}(\bar{w}-\tilde{x}) \\
& \hat{a}_{K i}=+\theta_{I i}{ }^{\sigma}(\bar{w}-\bar{x})
\end{aligned}
$$

onde $\Theta_{L i}$ e $\Theta_{K i}$ são as participações do trabalho e do capital na ren da total de cada setor i.

Substituindo agora essa solução para os coeficientes insumoproduto, nas equações (10) e (11), tem-se:

$$
\begin{aligned}
& \lambda I_{1} \mathrm{X}_{1}+\lambda \mathrm{I}_{2} \mathrm{~B}_{2}+\lambda \mathrm{L}_{\mathrm{N}} \mathrm{R}_{\mathrm{N}}=\tilde{E}+\mathrm{F}_{L} \cdot(\bar{w}-\hat{\mathrm{r}}) \\
& \lambda \mathrm{K}_{1} \mathrm{R}_{1}+\lambda \mathrm{K}_{2} \mathrm{R}_{2}+\lambda \mathrm{K}_{\mathrm{N}} \mathrm{g}_{\mathrm{N}}=\mathrm{R}-\mathrm{F}_{\mathrm{K}} \cdot(\hat{\mathrm{w}}-\overline{\mathrm{r}})
\end{aligned}
$$

onde

$$
\begin{aligned}
& F_{L}=\lambda I_{1} \Theta_{K 1} \sigma_{1}+\lambda I_{2} \Theta_{K 2} \sigma_{2}+\lambda L_{N} \theta_{K N} \sigma_{N} \geqslant 0 \\
& F_{K}=\lambda K_{1} \Theta_{L 1} \sigma_{1}+\lambda K_{2} \Theta_{L 2} \sigma_{2}+\lambda K_{N} \theta_{L N} \sigma_{N} \geqslant 0
\end{aligned}
$$


No caso de estar trabalhandc com coeficientes fixos de produção, $F_{L}$ e $F_{K}$ reduzem-se a zero. No caso que estamos considerando a qui, de coeficientes variāveis, ambos os termos são positivos.

Substituindo nas expressōes anteriores o valor correspondente a ( $(\hat{w}$ - $\hat{r})$ da equação (19), admitindo que a taxa de crescimento das dotaçōes de insumos seja nula e resolvendo tem-se:

$$
\frac{\mathrm{R}_{1}}{\left(\mathrm{~s}_{1}-\mathrm{s}_{2}\right)}=\frac{\mathrm{R}_{\mathrm{N}}\left|\mathrm{d}_{3}\right|}{\left|\mathrm{d}_{4}\right|\left(\mathrm{s}_{1}-\mathrm{E}_{2}\right)}+\left(\frac{\lambda \mathrm{K}_{2} \mathrm{~F}_{\mathrm{L}}+\lambda \mathrm{L}_{2} \mathrm{~F}_{\mathrm{K}}}{\left|\mathrm{d}_{4}\right|\left|\mathrm{d}_{1}\right|}\right)
$$

e

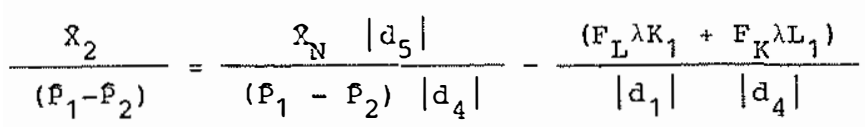

sendo

$$
\begin{aligned}
\left|\mathrm{d}_{3}\right|=\lambda \mathrm{K}_{\mathrm{N}} \lambda \mathrm{L}_{2}-\lambda \mathrm{K}_{2} \lambda \mathrm{L}_{\mathrm{N}} & <0 \text { se } \mathrm{k}_{\mathrm{N}<\mathrm{k}_{2}}^{>} \\
\left|\mathrm{d}_{4}\right|=\lambda \mathrm{K}_{2} \lambda \mathrm{L}_{1}-\lambda \mathrm{K}_{1} \lambda \mathrm{L}_{2} & <0 \text { se } \mathrm{k}_{2}{ }_{2} \mathrm{k}_{1} \\
\left|\mathrm{~d}_{5}\right|=\lambda \mathrm{K}_{1} \lambda \mathrm{L}_{\mathrm{N}}-\lambda \mathrm{K}_{\mathrm{N}} \lambda \mathrm{L}_{1} & <0 \text { se } \mathrm{k}_{1}>\mathrm{k}_{\mathrm{N}} \\
\text { e } \lambda \mathrm{K}_{2} \mathrm{~F}_{\mathrm{L}}+\lambda \mathrm{L}_{2} \mathrm{~F}_{\mathrm{K}} & >0 \\
\lambda \mathrm{L}_{1} \mathrm{~F}_{\mathrm{K}}+\lambda \mathrm{K}_{1} \mathrm{~F}_{\mathrm{L}} & >0
\end{aligned}
$$

3.1 0 Papel das Intensidades de Fatores e da Demanda Doméstica

As equaçōes (26) e (27) expressam as elasticidades termos de troca da produção de bens comerciāveis em função das relaçōes capi tal-trabalho nos três setores e da elasticidade termos de troca dos bens não-comerciāveis. Este è o aspecto novo, neste tipo de modelo com bens não-comerciāveis ${ }^{(9)}$. O efeito de mudanças nos termos de troca sobre a produção de bens comerciāveis depende não somente da estrutura tecnológica, mas também das condiçōes de demanda por bens 
domésticos. Assim, para conhecer os padrōes de produção na economia, é preciso que as condiçōes de demanda para os bens domésticos sejam consideradas explicitamente. E necessārio, portanto, explicitar equaçōes adicionais acerca do comportamento dos bens domésticos para resolver o sistema. Este problema é resolvido se levarmos em conta a equação de demanda por bens domésticos (3), da seção anterior. Diferenciando a equação (3) e expressando em termos de elasticidade temos:

$$
B_{N}=\left(P_{1}-P_{2}\right) \frac{E D_{N}}{E\left(P_{1} / P_{2}\right)}+\left(P_{N}-P_{2}\right) \frac{E D_{N}}{E\left(P_{N} / P_{2}\right)}+\hat{y} \frac{E D_{N}}{E Y}
$$

sendo

$$
\frac{E D_{N}}{E\left(P_{N} / P_{2}\right)}, \frac{E D_{N}}{E\left(P_{1} / P_{2}\right)} \text { e } \frac{E D_{N}}{E y}
$$

as elasticidades-preço direta, preço cruzada e renda, respectivaren te, da demanda por bens domésticos.

A expressão (29), entretanto, é uma equação em três variãveis: $\mathrm{X}_{\mathrm{N}},\left(\mathrm{P}_{\mathrm{N}}-\mathrm{P}_{2}\right)$ e $\hat{y}$. Assim, para determinar o sistema de equaçōes (26), (27) e (29) é preciso introduzir novas equaçōes.

Consideremos a renda real expressa na seguinte forma:

$$
\frac{Y}{P_{2}}=\frac{P_{1}}{P_{2}} \cdot x_{1}+x_{2}+\frac{P_{N}}{P_{2}} \cdot x_{N}
$$

Diferenciando essa expressão e considerando que neste modelo é vãlida a seguinte relação de produção geral (10) :

$$
\frac{\mathrm{P}_{1}}{\mathrm{P}_{2}} \cdot \mathrm{dx}_{1}+\mathrm{dx}_{2}+\frac{\mathrm{P}_{\mathrm{N}}}{\mathrm{P}_{2}} \cdot \mathrm{dx}_{\mathrm{N}}=0
$$

então, da expressão anterior fj.ca:

$$
\bar{Y}=\frac{P_{1} X_{1}}{Y}\left(s_{1}-s_{2}\right)+\frac{P_{N} X_{N}}{Y}\left(s_{N}-s_{2}\right)
$$


Dessa forma, as equaçōes (26), (27), (29), (30) e (21) formam un sistema determinado nas seguintes variāveis: $\mathrm{R}_{1}, \mathrm{R}_{2}, \mathrm{R}_{\mathrm{N}}, \overline{\mathrm{y}}$ e $\left(\mathrm{P}_{\mathrm{N}}-\mathrm{P}_{2}\right)$. Com base nesse sistema podemos examinar os efeitos de alteraçōes nos termos de troca sobre a produção de bens.

Substituindo a expressão (21) na equação (30) e logo em (29) e transformando, tem-se:

$$
\begin{gathered}
\frac{\mathrm{A}_{\mathrm{N}}}{\left(\mathrm{P}_{1}-\mathrm{P}_{2}\right)}=\frac{\mathrm{ED}_{\mathrm{N}}}{E\left(\mathrm{P}_{1} / \mathrm{P}_{2}\right)}-\frac{\left|\mathrm{d}_{2}\right|}{\left|\mathrm{d}_{1}\right|}\left[\frac{\mathrm{ED}_{\mathrm{N}}}{\mathrm{E}\left(\mathrm{P}_{\mathrm{N}} / \mathrm{P}_{2}\right)}+\frac{\mathrm{P}_{\mathrm{N}} \mathrm{X}_{\mathrm{N}}}{\mathrm{Y}} \frac{\mathrm{ED}_{\mathrm{N}}}{\mathrm{E} Y}\right]+ \\
+\frac{\mathrm{P}_{1} \mathrm{X}_{1}}{\mathrm{Y}} \cdot \frac{\mathrm{ED}_{\mathrm{N}}}{\mathrm{EY}}
\end{gathered}
$$

Utilizando a equação de slutsky, expressa em termos de elasti cidade, podemos simplificar a expressão (31) e obter:

$$
\frac{\mathrm{R}_{N}}{\left(\mathrm{P}_{1}-\mathrm{P}_{2}\right)}=\frac{E D_{N}}{E\left(P_{1} / P_{2}\right)}-\frac{\left|d_{2}\right|}{\left|\mathrm{d}_{1}\right|} \cdot \frac{E d_{N}}{E\left(P_{N} / P_{2}\right)}+\frac{\mathrm{P}_{1} X_{1}}{E_{Y}} \cdot \frac{E D_{N}}{E y}
$$

sendo $\frac{\mathrm{Ed}_{\mathrm{N}}}{\mathrm{E}\left(\mathrm{P}_{\mathrm{N}} / \mathrm{P}_{2}\right)}$ a elasticidade-preço da curva da demanda compen sada por bens domésticos. O valor desta elasticidade é sempre menor ou igual a zero.

Substituindo agora a equação (32) em (26) e (27) temos:

$$
\begin{aligned}
& \frac{8_{1}}{\left(P_{1}-P_{2}\right)}=\frac{\left|d_{3}\right|}{\left|d_{1}\right|}\left[\frac{E D_{N}}{E\left(P_{1} / P_{2}\right)}-\frac{\left|d_{2}\right|}{\left|d_{1}\right|} \frac{E d_{N}}{E\left(P_{N} / P_{2}\right)}+\frac{P_{1} X_{1}}{y} \frac{E D_{N}}{E y}\right]+ \\
& +\frac{\left(\lambda \mathrm{K}_{2} \mathrm{~F}_{\mathrm{L}}+\lambda \mathrm{L}_{2} \mathrm{~F}_{\mathrm{K}}\right)}{\left|\mathrm{d}_{4}\right|\left|\mathrm{d}_{1}\right|}
\end{aligned}
$$

e

$$
\begin{gathered}
\frac{\mathrm{B}_{2}}{\left(\mathrm{P}_{1}-\mathrm{P}_{2}\right)}=\frac{\left|\mathrm{d}_{5}\right|}{\left|\mathrm{d}_{4}\right|}\left[\frac{\mathrm{ED}_{\mathrm{N}}}{\mathrm{E}\left(\mathrm{P}_{1} / \mathrm{P}_{2}\right)}-\frac{\left|\mathrm{d}_{2}\right|}{\left.\left|\mathrm{d}_{1}\right| \frac{\mathrm{Ed}_{\mathrm{N}}}{E\left(\mathrm{P}_{\mathrm{N}} / \mathrm{P}_{2}\right)}+\frac{\mathrm{P}_{1} \mathrm{X}_{1}}{\mathrm{Y}} \frac{\mathrm{ED}_{\mathrm{N}}}{\mathrm{E} \mathrm{Y}_{-}}\right]-}\right. \\
-\frac{\left(\lambda \mathrm{L}_{1} \mathrm{~F}_{\mathrm{K}}+\lambda \mathrm{K}_{1} \mathrm{~F}_{\mathrm{L}}\right)}{\left|\mathrm{d}_{4}\right|\left|\mathrm{d}_{1}\right|}
\end{gathered}
$$


As expressões (32), (33) e (34) mostram que o efeito de umamu dança nos termos de troca externos sobre a produção dos bens depen de de uma série de parâmetros, os quais devem ser estudados. Diver sos casos podem surgir, dependendo das intensidades fatoriais e do valor da elasticidade-preço cruzada e renda da demanda por bens do mésticos. Todas as alternativas possíveis foram examinadas. Na Tabela 2 , a seguir, apresentaremos um resumo daqueles casos onde 0 efeito de uma melhoria nos termos de troca sobre a produção de bens seja bem definido. Nos demais casos (por exemplo, quando existe cam plementariedade bruta entre o bem doméstico e o exportável, e o bem doméstico não é inferior), o efeito sobre a produção de bens não é bem definido. Nesses casos, o efeito final fica dependendo do valor dos parâmetros.

Tabela 2

EFEITO DE UHA MELHORIA NOS TERMOS DE TROCA SOBRE A PRODUCAO DE BENS

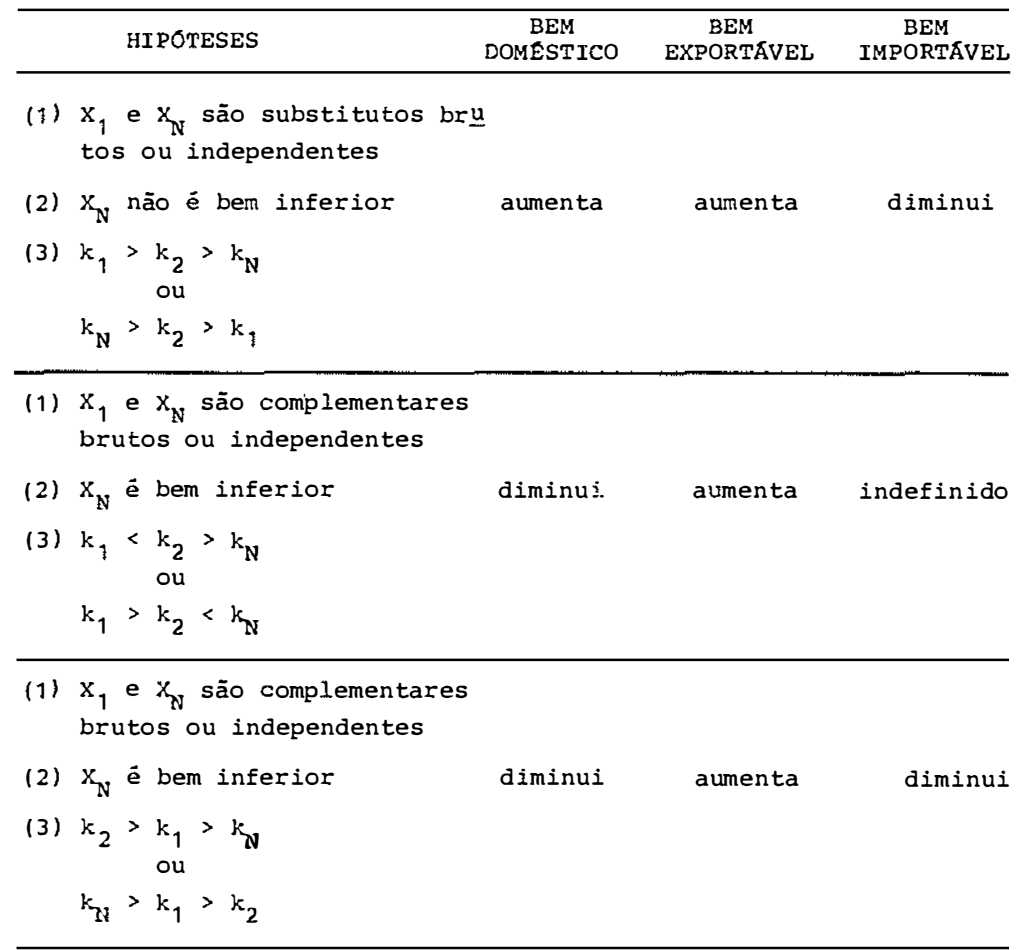


4. CONCLUSÕES

Este trabalho teve por objetivo analisar como os preços $e$ a produção de bens no mercado interno reagem às alterações nos termos de troca do comércio internacional aos incentivos e subsidios à exportação. Mostramos que o efeito de uma modificação nos termos de troca externos sobre o preço real do bem doméstico depende apenas da ordenação das intensidades fatoriais vigorando em cada um dos setores em que foi dividida a economia. Uma melhoria nos termos de troca leva a um aumento no preço real do bem doméstico somente quando o setor produtor de bens substitutos de importáveis é o mais intensivo em capital (trabalho) dos três setores considerados. Se as intensidades fatoriais estiverem ordenadas em forma diferente, então o preço real do bem doméstico diminui. Mostramos que essa conclusão independe de estarmos trabalhando com coeficien tes de produção fixos ou variāveis. O efeito de um incentivo ou subsídio no setor de bens exportáveis sobre o preço real do bem do méstico depende da mesma condição. A evidência empirica existente sugere que no caso do Brasil o setor produtor de bens substitutos de importáveis é o mais intensivo em capital dos três setores considerados. Espera-se, portanto, que no caso do Brasil uma melhoria nos termos de troca externos aumente o preço real dos bens doméstí cos.

Mostramos também que o efeito de uma mudança nos termos de troca externos sobre a quantidade produzida de bens depende não apenas das intensidades fatoriais, mas também das preferências dos consumidores. Para se conhecer os padrões de produção de bens comerciáveis na economia, é preciso que as condições de demanda para os bens domésticos sejam consideradas explicitamente. Uma melhoria nos termos de troca sempre aumentará a produção do bem exportá vel e diminuirá a produção do bem doméstico, desde que admitamos que o bem doméstico e o bem exportável são complementares brutos (ou independentes), sendo o bem doméstico inferior e o setor impor tador o mais intensivo em capital (trabalho) dos três setores da economia. Os resultados parecem indicar que substituibilidade bruta (complementariedade bruta) na demanda dos consumidores implica também substituibilidade bruta (complementariedade bruta) na produ cão dos bens. 
o objetivo deste apēndice é ampliar o modelo e examinar quais são os efeitos de mudanças exógenas nas dotações de fatores sobre a produção de bens na economia. Analisemos primeiro o efeito sobre a produção de bens domésticos. Admitindo a hipótese de equilíbrio competitivo, da equação (1) temos que:

$$
Y=W L+r K
$$

Diferenciando totalmente essa equação e expressando em termos de taxas de variação tem-se:

$$
Q=\theta_{L} \tilde{L}+\theta_{K} R+\theta_{L} \vec{w}+\theta_{K} \vec{r}
$$

Admitindo que os preços são mantidos constantes e substituindo (35) em (29) temos:

$$
\delta_{N}=\left(\theta_{L} f+\theta_{K} R\right) \cdot \frac{E_{N}}{E y}
$$

Assim da equação (36) conclui-se que excluindo o caso dos bens inferiores um aumento das dotações sempre incrementará a produção do bem doméstico. Este é um resultado já obtido por Komiya (1967), e é válido qualquer que seja a direção da produção dos bens internacionais.

A seguir examinaremos o efeito de mudanças nas dotações sobre a produção de bens exportáveis e importāveis. Veremos que os efeitos sobre a produção de bens comerciāveis são mais difíceis de serem interpretados. Não temos a pretensão de apresentar proposições simples e claras. Vamos apenas mostrar quais os fatores e parâmetros que devemos considerar na análise para chegar a conclusões 
definitivas. Veremos que a generalização do Teorema de Rybczynski com bens domésticos, fica a depender da elasticidade renda da demanda por bens não-comerciāveis e da intensidade fatorial nesse se tor.

Mantendo os termos de troca constantes, substituindo a equação (36) nas equações $\left(10^{\prime}\right)$ e $\left(11^{\prime}\right)$ e fazendo algumas transformações pode-se chegar à seguinte expressão:

$$
\begin{aligned}
\left|d_{4}\right|\left(R_{1}-R_{2}\right) & =\left[L\left(1-\lambda K_{N}\right)+\left(L \theta_{L}+R \theta_{K}\right) \frac{E D_{N}}{E y} \lambda K_{N}\left(1-\lambda L_{N}\right)\right]- \\
& -\left[R\left(1-\lambda L_{N}\right)+\left(E \theta_{L}+R \theta_{K}\right) \frac{E D_{N}}{E Y} \lambda L_{N}\left(1-\lambda K_{N}\right)\right]
\end{aligned}
$$

Conclui-se que $\delta_{1}>\delta_{2}$ somente se:

(a) $\left|\mathrm{d}_{4}\right|<0$; ou seja se $\mathrm{k}_{2}<\mathrm{k}_{1}$, e

(b) $\quad L\left(1-\lambda K_{N}\right)+\left(L \Theta_{L}+R \Theta_{K}\right) \frac{E D_{N}}{E y} \lambda K_{N}\left(1-\lambda L_{N}\right)<R\left(1-\lambda L_{N}\right)+$

$$
+\left(\mathrm{E} \Theta_{\mathrm{L}}+\mathrm{R} \odot_{\mathrm{K}}\right) \frac{\mathrm{ED_{N }}}{\mathrm{EY}} \lambda \mathrm{L}_{\mathrm{N}}\left(1-\lambda \mathrm{K}_{\mathrm{N}}\right)
$$

Fazendo algumas operações a condição (b) pode transformar-se em:

$$
\frac{L}{R}<\frac{\left(1-\lambda L_{N}\right)+\frac{E D_{N}}{E y} \odot_{K}\left(\lambda L_{N}-\lambda K_{N}\right)}{\left(1-\lambda K_{N}\right)+\frac{E D_{N}}{E y}\left(\Theta_{K}-1\right)\left(\lambda L_{N}-\lambda K_{N}\right)}
$$

Dessa forma, o efeito de mudanças nas dotações de fatores sobre a produção dos bens comerciāveis depende de uma série de parâmetros, os quais devem ser analisados. Chamemos o termo do lado direito da expressão (38) de $\emptyset$. Diversos casos surgem dependendode se $\emptyset$ é igual ou diferente da unidade. Conforme demonstrou Ethier (1972), se $\emptyset=1$ então conclui-se que:

Se $\mathrm{L}_{<}<\mathrm{R}$, então $\mathrm{X}_{1}>\mathrm{R}_{2}$ somente se $\mathrm{k}_{2}<\mathrm{k}_{1}$ e $\frac{\mathrm{ED}_{\mathrm{N}}}{\mathrm{Ey}}=1$ ou $\mathrm{k}_{\mathrm{N}}=\mathrm{k}$. 
Se $\emptyset$ é diferente da unidade pode-se demonstrar que as seguintes duas proposições são vālidas:

\section{Proposição (1):}

Se $\mathrm{L}^{>} \varnothing R, \emptyset<1$ (ou seja $k_{N}>k$ e $\frac{E D_{N}}{E y}<1$ (ou $k_{N}<k$ e $\left.\frac{E D_{N}}{E y}>1\right)$ ), então $\mathrm{R}_{1}>\mathrm{R}_{2}$ somente se $\mathrm{k}_{1}<\mathrm{k}_{2}$.

Ethier (1972, p.1.39-144)

existe um número $\emptyset$ tal que, ainda se ambos os fatores mudam no mes mo sentido, a produção do bem que é mais intensivo no fator que au mentou relativamente, incrementará a produção relativamente à produção do outro bem comerciāvel, sempre que $R<\mathcal{L}$ e $R<\emptyset \mathrm{L}$ ou que $R>L$ e $R>\emptyset$ L" . A demonstração da proposição (1) é uma prova desse teorema, mas agora especificando exatamente quais são os valores de $\emptyset$ que garantem a generalização do teorema de Rybczynski em um modelo com bens domésticos. Os possíveis casos "perversos", de que fala Ethjer (1972, p.140), ficam fora da proposição (1).

Proposição (2):

Se $\mathrm{C}<\emptyset \mathrm{R}, \emptyset<1$ (ou seja $\mathrm{k}_{\mathrm{N}}<\mathrm{k}$ e $\frac{\mathrm{ED}_{\mathrm{N}}}{\mathrm{EY}}<1$ (ou $\mathrm{k}_{\mathrm{N}}<\mathrm{k}$ e $\frac{\mathrm{ED}_{\mathrm{N}}}{\mathrm{EY}}>$ $>1)$ ), então $\mathrm{R}_{1}>\mathrm{R}_{2}$ somente se $\mathrm{k}_{1}<\mathrm{k}_{2}$.

A diferença que existe entre as proposições (1) e (2) reside no fato de que na proposição (1) o teorema de Rybczynski sobre dotações de fatores continua valendo. Por outro lado, na proposição (2) O teorema de Rybczynski pode não ser vālido. O problema que surge aqui deriva do fato que: $L<\emptyset R$ e $\emptyset>1$, não implica necessariamente $R>L$ e $I>\emptyset R$ e $\emptyset<1$, não implica necessariamente $R<L$, que são condições necessārias para garantir a generalização do teorema de Rybczynski, nesses casos. A explicação para o surgimento desses ca sos incertos, em um modelo com bens domésticos, deriva do fato que, quando as dotações de fatores aumentam, alguma parte desse aumento é absorvido na produção de bens domésticos. Como mostramos acima quando aumentam as dotações a produção do bem doméstico aumenta ou diminui, dependendo apenas da elasticidade-renda da demanda pelo bem doméstico ser positiva ou negativa. Quando as taxas de cresci 
mento da produção de bens comerciāveis não correspondem às espera das, caso o teorema de Rybczynski fosse válido e dado o aumento ocorrido nas dotações, o setor de bens domésticos deve estar absor vendo uma boa parte do insumo que aumentou mais rapidamente.

\section{APENDICE 2}

o objetivo deste apêndice é considerar os efeitos do progresso técnico no modelo. Primeiro colocaremos as equações básicas do modelo levando em conta mudanças técnicas em cada setor. Veremos que os efeitos do progresso técnico guardam uma certa semelhança com aqueles fenômenos estudados anteriormente. O progresso técnico vem apenas reforçar os efeitos de mudanças nos termos de troca ou nos subsídios e incentivos à produção de bens.

O estudo dos efeitos do progresso técnico se inicia consideran do os coeficientes insumo-produto uma função do preço relativo dos fatores de produção e da mudança tecnológica, ou seja:

$$
a_{j i}=a_{j i}\left(\frac{w}{r}, t\right) \quad \begin{aligned}
& i=1,2, N \\
& j=L, K
\end{aligned}
$$

Diferenciando totalmente essa expressão e expressando em termos de taxas relativas de variação, temos:

$$
\hat{a}_{j i}=\hat{c}_{j i}-\widehat{b}_{j i}
$$

onde $\vec{c}_{j i}$ representa a mudança no coeficiente insumo-produto devido a uma mudança nos preços relativos dos fatores de produção, para uma dada tecnologia. Por outro lado $6_{j i}$ é a medida do progresso téc nićo que resulta em uma mudança em $\hat{a}_{j i}$, mantendo os preços relativos dos fatores constantes. 
Substituindo a expressão (39) nas equações bāsicas do modelo (12), (13) e (14), utilizando novamente (16), (17) e (18) e resolvendo, chegamos às novas equações de mudanças de preços:

$$
\begin{aligned}
& \Theta_{\mathrm{L} 1} \hat{\mathrm{w}}+\Theta_{\mathrm{K} 1} \hat{\mathfrak{r}}=\hat{\mathrm{B}}_{1}+\bar{\pi}_{1} \\
& \Theta_{\mathrm{L} 2} \hat{\mathrm{w}}+\Theta_{\mathrm{K} 2} \hat{\mathrm{r}}=\hat{\mathrm{B}}_{2}+\bar{\pi}_{2} \\
& \Theta_{\mathrm{LN}} \hat{\mathrm{w}}+\Theta_{\mathrm{KN}} \hat{\mathrm{r}}=\hat{\mathrm{B}}_{\mathrm{N}}+\bar{\pi}_{\mathrm{N}}
\end{aligned}
$$

onde $\bar{\pi}_{i}$ é a taxa de progresso técnico no setor $i$.

Em forma semelhante, substituindo a expressão (39) nas equações básicas (10) e (11) e resolvendo, chegamos às novas expressões das mudanças nas quantidades:

$$
\begin{aligned}
& \lambda I_{1} \mathrm{X}_{1}+\lambda \mathrm{L}_{2} \mathrm{X}_{2}+\lambda I_{N} \mathrm{X}_{\mathrm{N}}=\mathrm{L}+\bar{\pi}_{L}+\mathrm{F}_{L}(\hat{\mathrm{w}}-\hat{\mathrm{r}}) \\
& \lambda \mathrm{K}_{1} \mathrm{~A}_{1}+\lambda \mathrm{K}_{2} \mathrm{R}_{2}+\lambda \mathrm{K}_{\mathrm{N}} \mathrm{B}_{\mathrm{N}}=\mathrm{R}+\hat{\pi}_{\mathrm{K}}-\mathrm{F}_{\mathrm{K}}(\hat{\mathrm{w}}-\hat{\mathrm{x}})
\end{aligned}
$$

onde $\tilde{\pi}_{L}$ e $\tilde{\pi}_{K}$ representam a economia total no uso do fator trabalho e capital, respectivamente, como consequência do progresso técnico.

Resolvendo as expressões (12") a (14") da mesma forma como foi feito na seção 2 deste trabalho, podemos chegar à seguinte expres são:

$$
\left(\hat{P}_{N}-\hat{s}_{2}\right)=-\frac{\left|d_{2}\right|}{\left|d_{1}\right|}\left(\hat{P}_{1}-\vec{P}_{2}+\bar{\pi}_{1}-\bar{\pi}_{2}\right)+\bar{\pi}_{2}-\bar{\pi}_{N}
$$

A expressão anterior mostra o preço real do bem doméstico em função dos termos de troca externos, do progresso técnico e das in tensidades fatoriais. Diversos casos podem surgir, dependendo do valor de cada parâmetro. A seguir vamos considerar apenas aqueles casos onde a mudança no preço real do bem doméstico seja bem definida. Uma melhoria nos termos de troca externos e progresso técnico do tipo $\pi_{1} \geqslant \pi_{2} \geqslant \pi_{N}$, levam a um aumento no preço real do bem domés tico sempre que a ordenação das intensidades fatoriais seja como indicado na expressão (22). Por outro lado, se o progresso técnico 
é do seguinte tipo: $\hat{\pi}_{1} \geqslant \pi_{2} \leqslant \pi_{N}$ e as intensidades fatoriais estão ordenadas na seguinte forma: $k_{1}>k_{2}>k_{N}$ (ou $k_{N}>k_{2}>k_{1}$ ), então, podemos concluir que uma melhoria nos termos de troca leva necessariamente a uma diminuição no preço real do bem doméstico. Nos demais casos, o resultado final não é bem definido.

o estudo dos efeitos do progresso técnico sobre os níveis de produção na economia podem ser feitos utilizando procedimento seme lhante aquele apresentado na seção 3. Conforme mostra-se em Barran tes (1983a) p. 66, progresso técnico do tipo: $\hat{\pi}_{1} \geqslant \pi_{2} \geqslant \pi_{N}$ necessariamente diminui a produção do beln doméstico e aumenta a produção do bem exportável desde que admitamos que o setor importador é o mais intensivo em capital (trabalho) dos três setores da economia, o bem doméstico sendo inferior e o bem exportável e doméstico comple mentares brutos ou independentes. Por outro lado a mudança na produção do bem importāvel é indefinida.

NOTAS

1. Entre os trabalhos que chamam a atençāo para esses problemas cabe destacar os de J. R. Mendonça de Barros e D. Graham (1978), Mauricio Barata de Paula Pinto e J. R. Mendonça (1979), e as pesquisas de Fernando Homem de Melo. Ver por exemplo: F. B. Homem de Melo (1978). Joāo L. Mascolo e M. Sanchez de La Cal (1983), utilizando um modelo de dois setores para a agricultura brasileira, discutem a existēncia de uma dicotomia en tre produtos de mercado doméstico e produtos de mercado exte $\bar{I}$ no. Gervásio Castro (1983) apresenta uma resenha da literatu ra que examina as interrelações entre o setor externo a agricultura brasileira.

2. Ainda que trabalhos anteriores tenham examinado a interação en tre a agricultura de exportação e a agricultura voltada pará o mercado doméstico, os modelos utilizados não permitem exami nar a interação entre os dois setores agricolas, já mencionados, e o setor industrial. E claro que todas as interaçóes relevantes só podem ser estudadas no contexto de um modelo de equilibrio geral.

3. No Apēndice 1 examinamos os efeitos de mudanças nas dotações de fatores.

4. Quando um terceiro bem é incorporado a um modelo com dois setores (dois bens e dois fatores), surge uma indeterminação no sistema se todos os bens forem comerciáveis. 0 conjunto eficiente de produção torna-se uma superficie regrada, como mos- 
trou Melvin (1968). Ver também Komiya (1967). Samuelson (1953 -1954), examina o caso mais geral de $n$ bens e $r$ fatores de pro dução em relação com o teorema da igualaçäo internacional dos preços dos fatores.

5. Ao longo de todo este trabalho será utilizado o instrumental matemático desenvolvido e popularizado por R. W. Jones (1965), no seu conhecido artigo sobre modelos de equilibrio geral a dois setores.

6. Segundo Jones (1965) p. 560, esta propriedade é mais um exemplo do teorema Wong-Viner referente à relaçāo de tangēncia que existe entre a envoltória de uma familia de curvas e as curvas que toca. Ver P. A. Samuelson (1947) p. 34 a 36 para maio res detalhes sobre o significado desse teorema e suas aplicaçöes econômicas.

7. Observe-se que:

$$
\left|d_{1}\right|=\Theta_{L 1}-\Theta_{L 2} \equiv \Theta_{K 2}-\Theta_{K 1}
$$

e

$$
\left|\mathrm{d}_{2}\right|=\Theta_{\mathrm{L} 2}-\Theta_{\mathrm{LN}} \equiv \Theta_{\mathrm{KN}}-\Theta_{\mathrm{K} 2} \cdot
$$

8. Veja Barrantes (1983b). Esses resultados mostram que o pais exporta bens relativamente intensivos no fator abundante (o trabalho) e importa bens relativamente intensivos no fator es casso (o capital). Essa evidencia parece corroborar a validade da teoria do comércio internacional de Heckscher-Ohlin para o Brasil.

9. Em um modelo com dois bens e dois fatores, um incremento no preço relativo de um dos bens sempre provocará um aumento no nivel de produção desse bem e um decréscimo na produção do ou tro bem.

10. Jones (1969) p. 419 mostra que no caso de dois bens é vàlida a seguinte relação: $\mathrm{dx}_{1}+\left(\mathrm{P}_{2} / \mathrm{P}_{1}\right) \mathrm{dx_{2 }}=0$. Para o caso de $n$ bens e trēs fatores, Batra (1973) p. 83-84 mostra que a seguinte relação de produção geral é välida: dx $1+\left(\mathrm{P}_{2} / \mathrm{P}_{1}\right) \mathrm{dx}_{2}$ $+\left(\mathrm{P}_{3} / \mathrm{P}_{1}\right) \mathrm{dx}_{3}+\ldots+\left(\mathrm{P}_{\mathrm{n}} / \mathrm{P}_{1}\right) \mathrm{dx_{n }}=0$. Em Barrantes (1983a) p. 38-39 fazemos a demonstração para o caso de trēs bens e dois fatores. 


\section{B IBL I OGRAF I A}

BARRANTES HIDALGo, Alvaro. "Efeitos dos Termos de Troca Internacionais sobre o Mercado Interno Brasileiro: Uma Análise de Equi librio Geral", Tese de Doutorado apresentada a Departamento de Economia da Universidade de São Paulo, 1983a.

- "Testes Empiricos do Teorema de Heckscher-Ohlin para o Brasil: Resenha da Literatura e Novos Resultados", Trabalho apresentado no XI Encontro Nacional de Economia da ANPEC, Belém/ /Pará, Dezembro de 1983b, p. 474-496.

BATRA, Raveendra. "Studies in the Pure Theory of International Tra de" (St. Martin's Press, New York, 1973).

CASTRo DE REzENDE, Gervàsio. "Setor Externo e Agricultura", Revis ta Literatura Éconômica, vol. 5 nọ 3 (maio-junho 1983).

ETHIER, Wilfred J. "Nontraded Goods and the Heckscher-Ohlin Model" International Economic Review, vol. 13 no 1 (February, 1972) p. $132-147$.

HOMEM DE MELO, F. B. "Agricultura Brasileira, Incerteza e Disponi bilidade de Tecnologia", Tese de Livre Docéncia, FEA-USP, 1978.

JONES, Ronald. "The Structure of Simple General Equilibrium Models", The Journal of Political Economy, vol. LXXII no 6, 1965, p. 557-572.

"Tariffs and Trade in General Equilibrium: Comment", American Economic Review, 59 (1969) p. 418-424.

KOMIYA, Ryutaro. "Non-Traded Goods and the Pure Theory of Interna tional Trade", International Economic Review, vol. 8 no 2 (june 1967) p. 132-152.

MASColo, J. L. e Manuel Sanchez de La Cal, "Anälise da Dicotomia entre Produtos Primários de Mercado Interno e Produtos de Expor tação", FUNCEX, Rio de Janeiro, 1983.

MELVIN, J. R. "Production and Trade With Two Factors and Three Goods", American Economic Review, 59 (December 1968) p. 1249-1268 .

MENDONCA DE BARROS, J. R. e D. Graham. "Agricultura Brasileira e o Problema da Produção de Alimentos", Pesquisa e Planejamento E conômico, Dez. 1978 . 
PINTo, Mauricio B. de Paula e José R. Mendonça de Barros. "A Agri cultura Brasileira e o Problema da Produção de Alimentos: Uma Exposiçào Gräfica", Revista Brasileira de Economia, vol. 33 no 1 (janeiro-março 1979 ).

SAMUElson, Paul Anthony. "Foundations of Economic Analysis", Cambridge, Harvard University Press, 1947.

"Prices of Factors and Goods in General Equilibrium", Review of Economic Studies, vol. 21 (1953-1954). 
